\title{
Malaria parasites form filamentous cell-to-cell connections during reproduction in the mosquito midgut
}

\author{
Ingrid Rupp ${ }^{1,}$, Ludmilla Sologub $^{1,}{ }^{*}$, Kim C Williamson ${ }^{2}$, Matthias Scheuermayer ${ }^{1}$, Luc Reininger ${ }^{3}$, \\ Christian Doerig ${ }^{3}$, Saliha Eksi ${ }^{2,6}$, Davy U Kombila ${ }^{4}$, Matthias Frank ${ }^{5}$, Gabriele Pradel $^{1}$ \\ ${ }^{I}$ Research Center for Infectious Diseases, University of Würzburg, Josef-Schneider-Strasse 2/D15, 97080 Würzburg, Germany; \\ ${ }^{2}$ Department of Biology, Loyola University Chicago, 6525 North Sheridan Road, Chicago, IL 60626, USA; ${ }^{3}$ INSERM U609, Global \\ Health Institute/Wellcome Trust Centre for Molecular Parasitology, Ecole Polytechnique Fédérale de Lausanne, GHI-SV-EPFL \\ Station 19, CH-1015 Lausanne, Switzerland; ${ }^{4}$ Medical Research Unit, Albert Schweitzer Hospital, Lambaréné, Gabon; ${ }^{5}$ Institute of \\ Tropical Medicine, University of Tübingen, Wilhelmstr 27, Tübingen 72074, Germany
}

Physical contact is important for the interaction between animal cells, but it can represent a major challenge for protists like malaria parasites. Recently, novel filamentous cell-cell contacts have been identified in different types of eukaryotic cells and termed nanotubes due to their morphological appearance. Nanotubes represent small dynamic membranous extensions that consist of F-actin and are considered an ancient feature evolved by eukaryotic cells to establish contact for communication. We here describe similar tubular structures in the malaria pathogen Plasmodium falciparum, which emerge from the surfaces of the forming gametes upon gametocyte activation in the mosquito midgut. The filaments can exhibit a length of $>100 \mu \mathrm{m}$ and contain the F-actin isoform actin 2 . They actively form within a few minutes after gametocyte activation and persist until the zygote transforms into the ookinete. The filaments originate from the parasite plasma membrane, are close ended and express adhesion proteins on their surfaces that are typically found in gametes, like $P f s 230, P f s 48 / 45$ or $P f s 25$, but not the zygote surface protein $P f s 28$. We show that these tubular structures represent long-distance cell-to-cell connections between sexual stage parasites and demonstrate that they meet the characteristics of nanotubes. We propose that malaria parasites utilize these adhesive "nanotubes" in order to facilitate intercellular contact between gametes during reproduction in the mosquito midgut. Keywords: malaria; nanotube; Plasmodium; gamete; fertilization; transmission; mosquito

Cell Research (2011) 21:683-696. doi:10.1038/cr.2010.176; published online 21 December 2010

\section{Introduction}

Cell-cell contact is an important mode of intercellular interactions in multicellular organisms as well as in communities of unicellular microbes. While adjacent cells in

*These two authors contributed equally to this work.

Correspondence: Gabriele Pradel

Tel: +49 931 3182174; Fax: +499313182578

E-mail: gabriele.pradel@uni-wuerzburg.de

${ }^{6}$ Present address: Department of Medical Microbiology, Rize University, Rize 53100, Turkey

Abbreviations: PC12 (pheochromocytoma cell); EM (erythrocyte membrane); FiGs (filaments of gametes); PVM (parasitophorous vacuole membrane); RT (room temperature).

Received 14 April 2010; revised 28 July 2010; accepted 12 September 2010; published online 21 December 2010 a tissue form features like gap junctions or synapses for the exchange of information, long distance communication is primarily mediated by the release of hormones or growth factors, without any physical contact between the communicating cells. Such exchange of soluble signaling molecules is particularly important for cells that are not embedded within a tissue, like bacteria or immune cells of higher eukaryotes.

A novel type of cell-to-cell connections was identified in 2004, which represents a transient filamentous structure between two interacting cells [reviewed in 1, 2]. These filaments were first described in rat pheochromocytoma (PC12) cells [3] and were later reported in a variety of mammalian immune cells, such as NK cells, dendritic cells, T cells and mast cells [4-8], thus cells that are either singular or form loose networks. Due to their morphological appearance they were termed membrane nano- 
tubes or tunneling nanotubes, respectively. Nanotubes can either form de novo by outgrowth of filopodia-like protrusions via actin polymerization [3] or they represent elongating membrane channels, which form as adhering cells move apart $[4,9]$. During recent years, extensive research on nanotubes has revealed that these structures represent a general mechanism for operational connectivity between cells [10], but instead of exerting a particular function, they reveal heterogeneity in their properties and have to be divided into subclasses [1,2].

Nanotubes are typically $50-200 \mathrm{~nm}$ in diameter and exhibit a length of up to $100 \mu \mathrm{m}$. Such filaments consist of F-actin, while both F-actin and microtubules can be found in so-called thick nanotubes, which are $>700 \mathrm{~nm}$ in diameter [9]. Nanotubes were reported to either display a continuous membrane between two connected cells, as described for PC12 and dendritic cells, or are close-ended, like T-cell-specific nanotubes [6]. Several functions were attributed to nanotubes, depending on the cell type, from which they originate. Assigned functions range from trafficking of vesicles or mitochondria, as described for PC12 cells and macrophages $[3,9,11]$ to mediating intercellular $\mathrm{Ca}^{2+}$ signaling, as was shown for myeloid cells [5].

We here report similar filamentous structures in the sexual stages of the human malaria parasite Plasmodium falciparum, which form in the mosquito midgut during reproduction. The sexual phase of the malaria parasite is initiated by the differentiation of intraerythrocytic sexual precursor cells in the human host, the gametocytes. Upon uptake by the blood-feeding female anopheline mosquito, a drop in temperature as well as factors in the mosquito midgut trigger the activation of gametocytes, resulting in their egress from the enveloping erythrocyte [reviewed in 12,13$]$. The activated female gametocytes round up and transform into macrogametes, while activated male gametocytes undergo three rounds of nuclear division and form eight flagella-like microgametes, which actively detach from the central body in a process called exflagellation and move freely in the medium before fertilizing a receptive macrogamete. Within a day post activation, the resulting zygote transforms into the infective ookinete, which is able to exit the midgut lumen by disrupting the midgut epithelium [reviewed in 12].

In this study, we describe that activated gametocytes develop membranous filaments by means of cell-derived surface extensions, which can form membranous bridges between gametes. The filaments display typical features of nanotubes and might represent a novel type of long distance cell-to-cell connections between the sexual stages of malaria parasites within the mosquito midgut.

\section{Results}

Gametocytes form membranous filaments following activation

We initially identified the filamentous cell-to-cell connections via immunolabeling of malaria sexual stage parasites. Immunofluorescence assays of gametocytes undergoing gametogenesis, using antibodies against the sexual stage surface protein $P f_{s} 230$ (Figure 1A), revealed thin tubular structures, which originate from the activated gametocytes upon rounding up and form bridges between these cells. These filaments were particularly evident in emerging macrogametes, as indicated by $P f_{s} 25$ labeling (Figure 1B), although similar structures were also observed in activated microgametocytes during exflagellation (see below). The length of the protrusions varied between 2 and $180 \mu \mathrm{m}$, with the average filament length varying between 10 and $20 \mu \mathrm{m}$ depending on the culture (examples given in Table 1). Up to 11 filaments were detected on the surface of gametes, which often exhibit bead-like structures in periodic intervals (shown in Figure 1B, 2F and 2K). No filaments were found in non-activated gametocytes (data not shown). Due to their morphological appearance, we henceforth will refer to these structures as "filaments of gametes" (FiGs).

Time-course studies showed that FiGs developed within a few minutes after activation of mature gametocytes (Figure 1C), coincident with the first appearance of axonemes in the exflagellating microgametocytes (Figure 1D). The data indicate that formation of these surface protrusions is initiated while the activated gametocyte is still in the process of egress, which takes $\sim 8 \mathrm{~min}$ [reviewed in 12]. As mentioned above, filaments were observed both in the emerging macrogametes as well as in the exflagellating microgametocytes, and at $7 \mathrm{~min}$ post activation, $\sim 27 \pm 2.9 \%$ of filament-forming microgametocytes versus $15 \pm 5.0 \%$ filament-forming macrogametes were observed $(n=20)$. Within a time period of $30 \mathrm{~min}, \sim 50 \%$ of gametes formed FiGs (Figure 1C). The filaments remained on the surface of macrogametes and zygotes at later time points (1-15 h post activation; Figure $1 \mathrm{C}$ ). Approximately $2 \mathrm{~h}$ post activation, the gametes were often detected to form cell clusters (Figure 1E). Up to eight macrogametes were observed in these clusters (an average of $3 \pm 1.6$, out of 30 clusters investigated), and cells within these clusters were connected by multiple FiGs, suggesting that the filaments exhibit adhesive properties. In some cases, cell clusters of $>20$ gametes were observed (Figure 1F). The filaments remain present on the surface of zygotes and retorts 1 day post activation. On retorts, which represent intermediate stages during the transformation of the zygote into the elongated ookinete, 

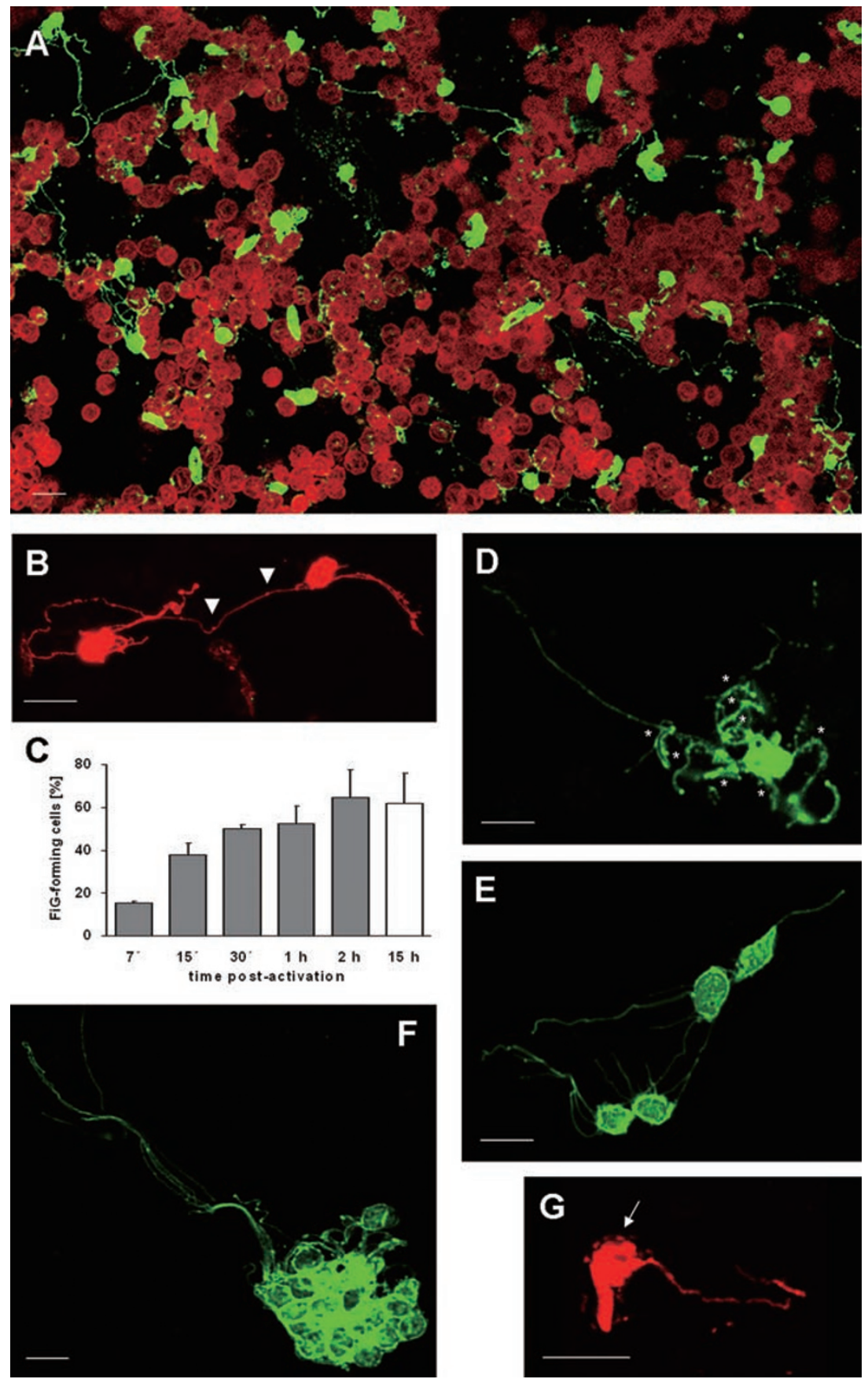

Figure 1 Malaria gametocytes form filamentous cell-to-cell connections upon activation. (A) Immunofluorescence assays on exflagellating cultures reveal thin tubular structures between emerging gametes on gametocyte activation, as shown by labeling with antibodies against Pfs230 (green). Erythrocytes were counterstained with Evans Blue (red). (B) Filamentous structures were further observed by immunolabeling of macrogametes with antibodies against Pfs25. Arrowheads indicate beadlike structures. (C) Time-course studies on exflagellating cultures showed $15 \%$ of gametocytes developing filaments within 7 min after activation, which increased to $\sim 50 \%$ of filament-positive cells being counted at $30 \mathrm{~min}$ and later time points. Data are represented as mean +/- SEM. Gray bar: in vitro activated cultures investigated via Pfs230-labeling; white bar: endogenously activated macrogametes within the mosquito midgut investigated via Pfs25-labeling. (D) Filaments first appear at $\sim 7$ min post activation, coincident with the emergence of microgametes (indicated by asterisks), as shown by immunolabeling with antibodies against Pfs230 (green). (E) At $2 \mathrm{~h}$ post-activation gametes had formed cell clusters connected by multiple cell filaments. (F) In some cases, gametes assembled to clusters of $>20$ cells. (G) Pfs25-labeling (red) demonstrates that filaments remained present on the spherical zygote-derived part of retort stages (arrow). For immunolabeling, if not noted otherwise, primary antibodies were used in combination with Alexa488 (green) or Alexa594 (red) secondary antibodies. Bar, $10 \mu \mathrm{m}$. 

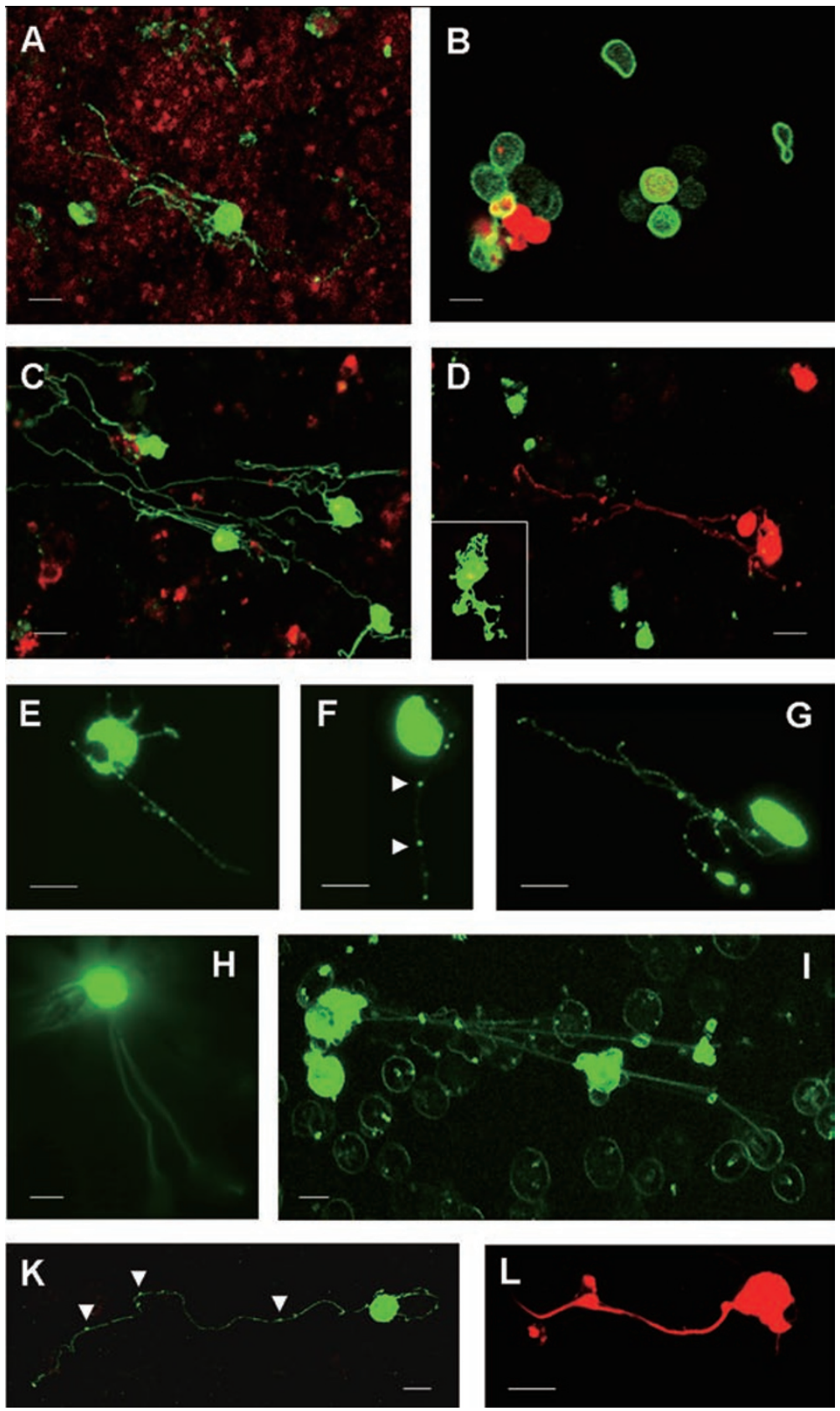

Figure 2 Filaments originate from the gamete surface and exhibit both cytoplasmic content and F-actin. (A) Gametocytes that were treated with saponin before activation to remove EM and PVM retain the ability to form filaments, as shown by immunolabeling of Pfs230 (green). Fragments of lysed erythrocytes were counterstained with Evans Blue (red). (B) No filaments were detected by immunolabeling of activated gametocyte cultures with antibodies against band 3, which reacts with the EM (green). Sexual stage parasites were identified by antibodies against $\alpha$-tubulin II (red). It is noteworthy that $\alpha$-tubulin II does not label filaments. (C) Pfs230-positive FiGs (green) are not labeled by antibodies against the PVM protein EXP-1 (red). (D) Immunolabeling also revealed that Pfs25-positive filaments (red) are not labeled by antibodies against the sexual stage PVM protein Pfs16 (green). However, in some cases antibodies against Pfs 16 reacted with filamentous and bleb-like structures in the emerging gametocytes (inset). (E-G) Filament formation was observed in live gametes of a parasite line episomally expressing GFP-tagged Pfnek-4 under the control of its cognate promoter. $(\mathrm{H})$ Similarly, the parasite line 5' CAM.GFP, which drives episomal expression of GFP by a calmodulin promoter, was used and revealed filaments protruding from the gamete surface. (I) Filament formation was further observed in gametes of LysoSensor-labeled live parasites. $(\mathbf{K}, \mathbf{L})$ The filaments contain F-actin, as indicated by labeling with anti-Pfact2 antibody (K) or by TRITC-phalloidin-labeling (L). Arrowheads indicate bead-like structures. For immunolabeling, if not noted otherwise, primary antibodies were used in combination with Alexa488 (green) or Alexa594 (red) secondary antibodies. Bar, $5 \mu \mathrm{m}$. 

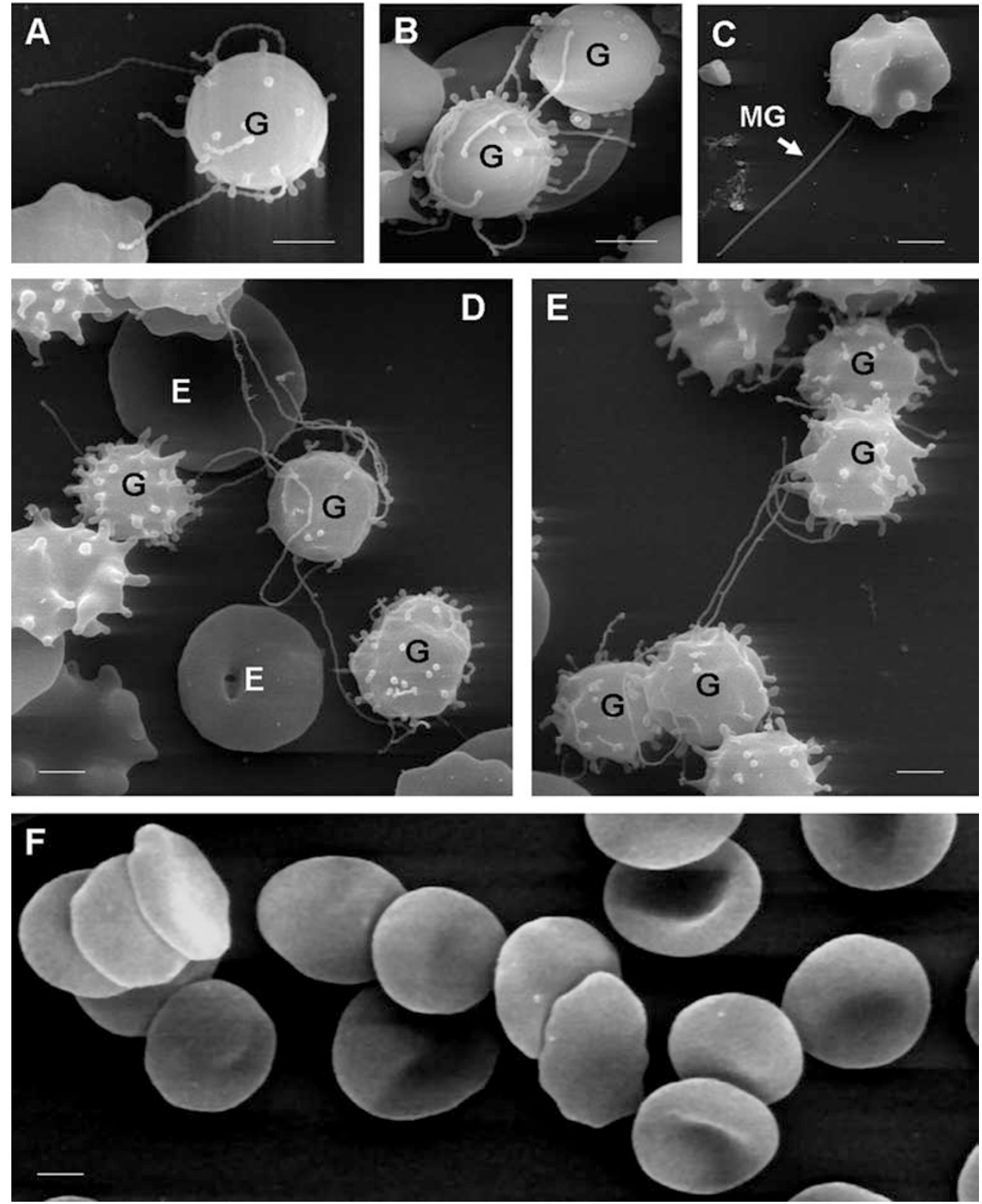

Figure 3 Multiple close-ended filaments originate from the gamete surface. (A, B) Scanning electron microscopy reveals multiple filaments originating from the parasite surface. The surface protuberances exhibit a beaded structure and appear to be close-ended. (C) Contrary to the appearance of the filaments, microgametes exhibit a smooth and even surface and a constant length of $\sim 15 \mu \mathrm{m}$. (D, E) The filaments primarily form cell-to-cell connections between gametes, but also adhere to erythrocytes. (F) No filaments were observed arising from the surface of uninfected erythrocytes. Arrow indicates a microgamete. E, erythrocyte; G, gamete; MG, microgamete. Bar, $2 \mu \mathrm{m}$.

they are associated with the spherical zygote-derived part, but not with the ookinete surface (Figure 1G).

Filaments are protrusions of the gamete plasma membrane

Unactivated gametocytes are surrounded by multiple membranes and, within minutes after activation, they emerge from both the parasitophorous vacuole membrane (PVM) and the erythrocyte membrane (EM) [reviewed in 13]. Rudiments of shed membranes can subsequently be observed adjacent to the newly formed gametes and around exflagellation centers, as shown by transmission electron microscopy (Supplementary information, Figure S1.) We therefore aimed at investigating the type 
of membrane from which the filaments derived. First, mature gametocytes were treated with saponin prior to activation. Saponin-treatment results in the loss of the enveloping EM as well as the PVM, but leaves the parasite membrane intact [14]. Filaments were still observed in gametes of saponin-treated cultures, as shown by the immunofluorescence assay (Figure 2A, Supplementary information, Figure S2). Furthermore, EM labeling in fixed gametocyte cultures $30 \mathrm{~min}$ post activation, using antibodies against band 3, did not highlight any filamentous structures (Figure 2B), indicating that the filaments do not originate from the EM.

We then investigated the potential role of the PVM in FiG formation in more detail. Immunofluorescence assays revealed that FiGs do not label for EXP-1, a transmembrane protein of the plasmodial PVM (Figure 2C) [15]. Further, no $P f \mathrm{~s} 16$, a protein associated with the gametocyte PVM [16, 17], was found in association with the FiGs (Figure 2D). Worth mentioning, $P f \mathrm{~s} 16$ labeling in some cases highlighted short filamentous and bleblike structures associated with activated gametocytes (Figure $2 \mathrm{D}$, inset), which was presumably caused either by fragments of the rupturing PVM or by partial adhesion of the protein to the gametocyte plasma membrane. The combined immunolabeling data let us conclude that the filaments are protrusions of the parasite surface and do not result from any other membranes that enveloped the gametocyte.

Scanning electron microscopy was performed on activated cultures to gain deeper insight into the morphology of FiGs, and it revealed that the structures are $\sim 200 \mathrm{~nm}$ in diameter. The filaments exhibit a beaded structure and appear to be close ended (Figure 3A, 3B). Microgametes, in comparison with FiGs, have a fixed length of $\sim 15 \mu \mathrm{m}$ as well as an even and smooth surface with pointed tips (Figure 3C). Multiple FiGs originated from the macrogamete surface and formed adhesive cell connections with other gametes (Figure 3D, 3E). No such filaments were observed in scanning electron micrographs of uninfected erythrocytes (Figure 3F).

The FiG ultrastructure was further investigated by transmission electron microscopy, which was a technically challenging procedure due to the two-dimensional limitation in ultrasections. Transmission electron micrographs confirmed that the FiGs represent protrusions of the gamete surface, which contain cytoplasmic content (Figure 4A-4C). Interestingly, electron dense plaques were in some occasions observed associated to the filament membranes (Figure 4B), which might represent accumulations of proteins and which give the filament surface an uneven shape. Transmission electron microscopy confirmed that microgametes are morphologically different from FiGs. Microgametes exhibit a smooth surface and axonemal microtubules are regularly detected in their inside (Figure 4D).

The FiGs were also investigated for the presence of surface-associated proteins by immunofluorescence assay, and shown to express adhesion proteins typical for gametes [reviewed in 12], like $P f \mathrm{~s} 25$ (Figure 1B) and $P f_{\mathrm{s}} 230$ (Figure 1A, 1D-1F), as well as $P f_{\mathrm{s}} 48 / 45$ and PfCCp4 (data not shown). Proteins that are typically expressed on the surface of gametocytes, but whose expression ceases during gametogenesis, e.g., PfPeg3 and PfPeg4 [18], were not present in the FiGs. Similarly, no $P f C C p 1, P f C C p 2$ and $P f C C p 3$ proteins were detected (data not shown). These proteins are highly expressed in gametocytes in association with the plasmalemma, but are partly released during emergence, and subsequently found on the macrogamete surface in a patchy pattern $[19,20]$. For an additional negative control, specimens were treated with antibodies against the endoplasmic reticulum-associated protein $P f 39$ [21], and no labeling of FiGs was observed (data not shown). Noteworthy, no $P f$ s 28 protein was found associated with the FiGs (data not shown). This protein was shown in the rodent malaria model to be translationally repressed in gametocytes and protein expression is then initiated at the time point when the zygote develops $[22,23]$.

Although the filament surface is covered with prominent gamete-associated adhesion proteins, these are not required for FiG formation, as was shown in three malaria parasite lines lacking these proteins. Filaments were observed in PfCCp1-KO parasites, which lack all of the $P f C C p$ proteins [24], in $P f s 230-\mathrm{KO}$ parasites, expressing a truncated form of the protein $[25,26]$, and in $P f_{s} 48 / 45-\mathrm{KO}$ parasites, lacking $P f_{S} 48 / 45$ and $P f_{S} 230[26,27]$ (Table 1). Notably, quantification of filaments revealed that FiG-formation in these lines was impaired (Table 1). We therefore investigated the ability of $P f s 230-\mathrm{KO}$ and $P f_{s} 48 / 45-\mathrm{KO}$ parasites to form cell clusters, and detected a reduction of clusters with high cell numbers at $2 \mathrm{~h}$ post activation. While a number between 2 and 20 cells with an average of $4 \pm 3.8$ cells per cluster were observed in wild-type parasites, between 2 and 4 cells with an average of $2 \pm 0.6$ cells per cluster were detected in the $P f s 230-\mathrm{KO}$ line and between 2 and 6 cells with an average of $3 \pm 1.1$ were detected in the $P f_{s} 48 / 45-\mathrm{KO}$ line (out of 30 cell clusters investigated; specimens labeled with anti$P f s 25$ antibody).

In accordance with the data on gene-disruptant lines, FiG formation cannot be inhibited by addition of antibodies against the prominent surface proteins $P f s 25$ and $P f s 230$ (Table 2). In the presence of $P f s 230$ antibodies, though, filaments appeared shorter than in the controls, 
which were treated either with neutral mouse serum or with buffer alone. The combined data indicate that the presence of surface-associated adhesion proteins is not required for the formation of filaments. However, filament number and length can be reduced when select filament-associated proteins are missing or blocked.
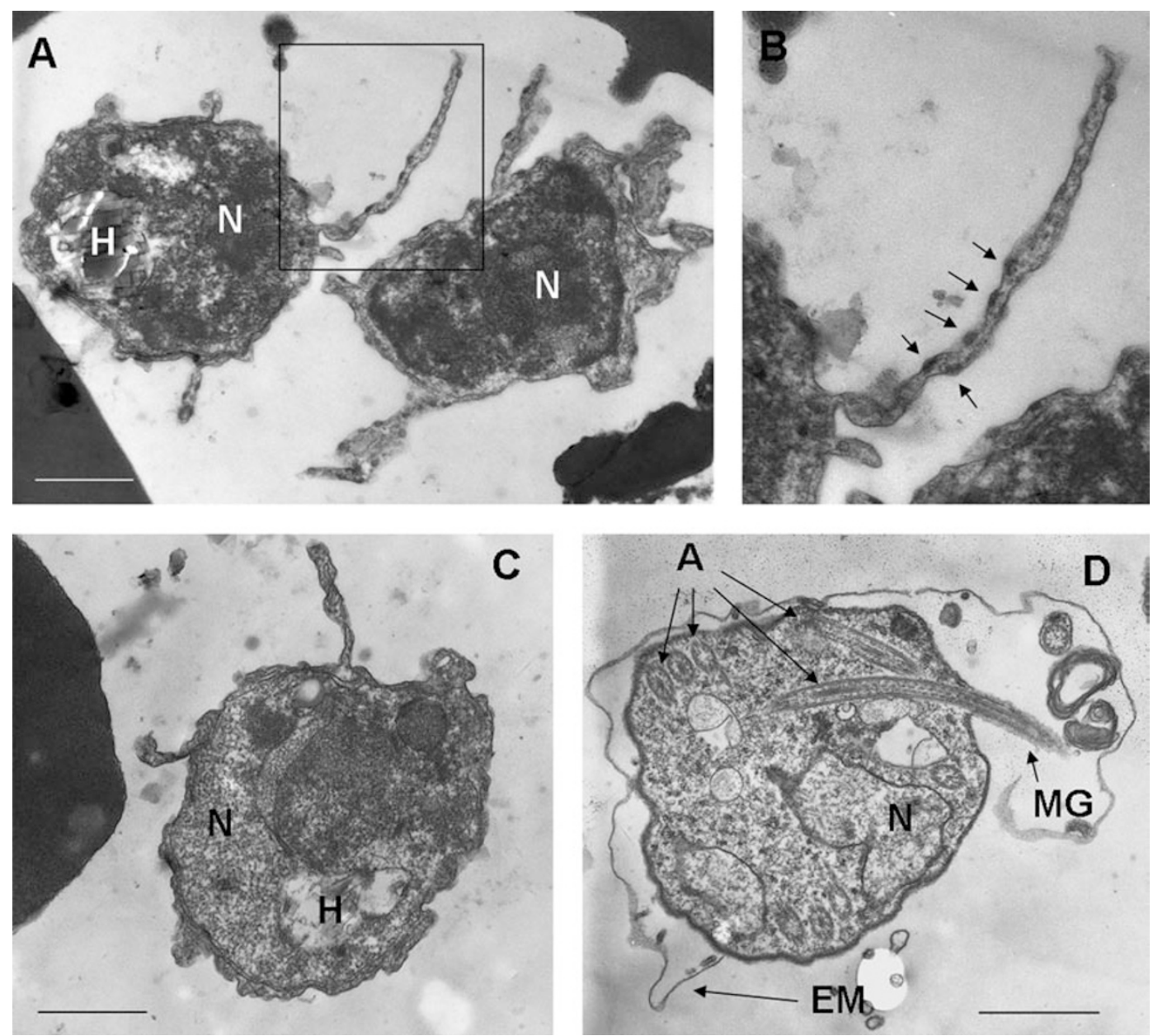

Figure 4 Filaments protrude from the gamete surface. (A-C) Transmission electron micrographs depict macrogametes, which form filamentous cytoplasmic extensions arising from their plasma membrane. Inset (A) indicates the area enlarged in (B). Arrows indicate electron-dense accumulations associated with the filament membrane, which result in an uneven structure of the filament surface. (D) Microgametes, which are formed by the activated microgametocyte, exhibit an even surface, and axonemal microtubules can be observed in their inside. A, axoneme; EM, erythrocyte membrane; H, hemozoin; MG, microgamete; N, nucleus. Bar, $2 \mu \mathrm{m}$.

Table 1 Quantification of FiGs in wild-type and gene-disruptant parasites

\begin{tabular}{|c|c|c|c|c|c|c|}
\hline & & $\mathrm{WT}^{*}$ & $\mathrm{WT} * *$ & PfCCp1-KO* & $P f_{\mathrm{S}} 48 / 45-\mathrm{KO}^{* *}$ & $P f \mathrm{~s} 230-\mathrm{KO}^{* *}$ \\
\hline \multirow[t]{3}{*}{ Culture 1} & Cells with FiGs (\%) & $54.0 \pm 2.00$ & $46.0 \pm 4.00$ & $34.0 \pm 3.46$ & $36.0 \pm 3.46$ & $30.7 \pm 3.06$ \\
\hline & No. of FiGs per cell (range) & $2.7(1-7)$ & $3.3(1-8)$ & $2.3(1-6)$ & $2.7(1-11)$ & $3.0(1-7)$ \\
\hline & Length ( $\mu \mathrm{m}$; range) & $19.3(2-120)$ & $13.7(2-140)$ & $13.3(2-90)$ & $17.3(2-140)$ & $21.7(2-180)$ \\
\hline \multirow[t]{3}{*}{ Culture 2} & Cells with FiGs (\%) & $55.3 \pm 7.57$ & & $32.7 \pm 2.31$ & $22.0 \pm 2.00$ & \\
\hline & No. of FiGs per cell (range) & $2.0(1-6)$ & & $2.0(1-10)$ & $2.3(1-8)$ & \\
\hline & Length ( $\mu \mathrm{m}$; range) & $18.0(2-120)$ & & $18.0(2-120)$ & $16.7(2-140)$ & \\
\hline
\end{tabular}

Detected by immunolabeling of $P f \mathrm{~s} 230\left(^{*}\right)$ or $P f \mathrm{~s} 25\left(^{* *}\right)$; for each condition 50 activated gametocytes were counted in triplicate. 
Table 2 Quantification of FiGs in antibody-treated cultures

\begin{tabular}{|c|c|c|c|c|c|c|c|}
\hline & & $\begin{array}{l}\text { Medium } \\
\text { control* }^{*}\end{array}$ & NMS* & $\begin{array}{l}P f_{\mathrm{s}} 230 \\
\text { antibody* }\end{array}$ & $\begin{array}{l}\text { Medium } \\
\text { control** }\end{array}$ & $\mathrm{NMS}^{* *}$ & $\begin{array}{l}P f \mathrm{~s} 25 \\
\text { antibody** }\end{array}$ \\
\hline \multirow[t]{2}{*}{ Culture 1} & Cells with FiGs (\%) & 40 & 42 & 40 & 52 & 60 & 48 \\
\hline & Length ( $\mu \mathrm{m}$; range) & $19.9(2-100)$ & $26.8(2-120)$ & $7.8(2-70)$ & $28.5(2-160)$ & $35.2(2-120)$ & $38.2(2-100)$ \\
\hline Culture 2 & Cells with FiGs (\%) & 34 & 38 & 40 & 58 & 56 & 52 \\
\hline
\end{tabular}

Detected by immunolabeling of $P f \mathrm{~s} 25\left(^{*}\right)$ or $P f \mathrm{~s} 230(* *)$; for each condition 50 activated gametocytes were counted. NMS, non-immunized mouse serum.

\section{Filaments form actively and contain F-actin}

FiGs were also observed in live P. falciparum cultures. In accordance with our data on fixed parasite cultures, live macrogametes forming FiGs were detected in two independent episomal GFP-expressing parasite lines, one expressing a GFP-tagged version of the female-specific $P f$ nek-4 NIMA-related kinase under the control of its cognate promoter (Figure 2E-2G) [28] and the other one in which GFP expression was driven by a calmodulin promoter (Figure 2H) [29]. These experiments demonstrate that FiGs are produced by live parasites and that they contain cytoplasmic content. In another approach, we incubated exflagellating cultures with the cell dye LysoSensor, which resulted in intense labeling of acidic compartments, and also in a fluorescent background labeling of the parasite cytoplasm, but not the erythrocyte cytoplasm. Again FiG labeling was detected (Figure 2I). When observed under the microscope, active movement was monitored and the result showed that the filaments can be actively pulled in (Supplementary information, movie S1).

To investigate, if FiGs represent active protrusions or if they are pulled out by an adjacent cell during divergence, we analyzed FiG formation in activated cultures, which exhibited different numbers of gametocytes at a constant number of total cells per volume. Quantifica-

Table 3 Quantification of FiGs in activated gametocyte cultures with decreasing parasite concentrations

\begin{tabular}{llccc}
\hline & & Undiluted & $\begin{array}{c}1: 2 \\
\text { dilution }\end{array}$ & $\begin{array}{c}1: 10 \\
\text { dilution }\end{array}$ \\
\hline Culture 1 & Cells with FiGs (\%) & 44 & 40 & 46 \\
Culture 2 & Cells with FiGs (\%) & 42 & 46 & $48^{*}$ \\
\hline
\end{tabular}

Detected by immunolabeling of $P f_{\mathrm{s}} 230$; a constant number of 11.1

$\times 10^{6}$ erythrocytes total (uninfected + infected) were used per $200 \mu \mathrm{l}$ sample; for each condition 50 or $25\left(^{*}\right)$ activated gametocytes were counted. tion of FiG-forming gametes in these cultures by $P f s 230$ immunolabeling revealed no differences in the number of filament-positive cells compared with the total number of gametes, which was $\sim 45 \%$ (Table 3 ). These data indicate that filament formation is independent from the chance of a given gamete to have come in contact with another gamete. Since filaments are sometimes found adhering to erythrocytes, we further investigated, whether binding of gametes to the erythrocyte surface might be the cause of FiG formation. Gametocyte cultures were therefore treated with chymotrypsin in order to remove glycophorins on the surface of erythrocytes prior to activation. In chymotrypsin-treated activated cultures FiGs were observed, which did not reveal any morphological difference to the ones formed in untreated activated cultures (Supplementary information, Figure S3A).

We further wanted to investigate the presence of actin or microtubules in these structures. Previous studies have assigned $\mathrm{F}$-actin to nanotubes of higher eukaryotic cells [reviewed in 1, 2]. In Apicomplexa, however, the majority of actin exists as the monomeric form rather than being polymerized to F-actin $[30,31]$. The $P$. falciparum genome encodes for two actin genes, termed Pfact1 (PFL2215w) and Pfact2 (PF14_0124) [32], the latter being predominantly expressed in the sexual stages $[33,34]$. For our studies, we generated polyclonal antisera against $P$ fact 2 in mice and characterized $P$ fact 2 expression in sexual stage parasites via immunofluorescence assay and western blot analysis. The antisera led to an intense labeling of gametocytes (stages III to V; Supplementary information, Figure S4A) as well as of macrogametes and microgametes, which were identified by double labeling with antibodies against $P f s 25$ and the male-specific $\alpha$-tubulin II [35], respectively (Supplementary information, Figure S4B, S4C). In western blot analysis, Pfact2 antisera reacted with a protein band running at the expected molecular weight of $\sim 43 \mathrm{kDa}$ in lysates of mature gametocytes and of gametocytes $15 \mathrm{~min}$ post 
activation (Supplementary information, Figure S4D). An additional protein band at $\sim 70 \mathrm{kDa}$ was also detected. Faint protein bands were further detected in lysates of the gametocyte-less F12 strain, indicating that Pfact2 is also present in asexual blood stages at a low expression level. No protein band was detected in control lysates of uninfected erythrocytes (Supplementary information, Figure S4D). Further, no bands were detected in parasite lysates, when sera of non-immunized mice were used (data not shown).

Immunofluorescence assays demonstrated that $P f$ act 2 is also present in the filamentous structures (Figure $2 \mathrm{~K}$; Supplementary information, Figure S4A and S4B, S5A). As a second method to demonstrate actin within FiGs, we used TRITC-labeled phalloidin for immunodetection. Phalloidin binds at the interface of the F-subunits, thereby stabilizing the actin filaments, and would recognize both $P$ fact1 and Pfact2. TRITC-labeled phalloidin highlighted asexual parasites as well as gametocytes and gametes (data not shown) and also labeled the FiGs (Figure 2L). Microtubules, on the other hand, were not detected in association with FiGs, when parasites were labeled with antibodies against $\alpha$-tubulin (Supplementary information, Figure S5B) or $\alpha$-tubulin II (Supplementary information, Figure S4B).

To investigate the effect of actin inhibitors on FiG formation, we treated activated cultures with cytochalasin $\mathrm{B}$, which interferes with actin monomer polymerization. Cytochalasin B treatment significantly reduced the number of FiG-forming cells by $33 \%$, as indicated by $P f_{S} 230$ labeling of activated gametocyte cultures $(P<0.05$, Student's $t$-test).

Filaments are formed by viable gametes in the mosquito midgut

In a final set of experiments we wanted to confirm that FiGs are indeed cell structures of viable parasites, but not artificially induced by in vitro cultivation. First, we investigated a $P$. falciparum field isolate, 166MOA, which was isolated from a male patient in Gabon $\sim 4$ month prior to the experiments. Macrogametes of the field isolate formed FiGs following activation of gametocytes, similar to the ones described for the laboratory strain NF54, as shown by immunofluorescence assay using anti- $P f s 25$ antibodies (Supplementary information, Figure S3B). In a second set of experiments we fed Anopheles stephensi mosquitoes with non-activated mature gametocyte cultures. Live imaging of midgut smears $\sim 10$ min post feeding showed the formation of FiGs by macrogametes (Supplementary information, Figure S3C). Similarly, immunofluorescence assays on fixed midgut smears revealed FiGs in the blood meal, when these were labeled with anti-Pfs 25 antibodies (Supplementary information, Figure S3D). These results indicate that FiGs are naturally produced structures of malaria parasites, which are utilized by the parasite sexual stages.

\section{Discussion}

The formation of filamentous cell protrusions was described in protozoa as early as the 70 s of last century, like the highly adhesive thread-like filopodia arising from the surface of trypanosomes [e.g., 36-38]. Back then, these filaments were assigned to reduced mobility of the parasites [38] and later fell into oblivion. The recent discovery of filopodia-like cell protrusion as a novel form of intercellular communication in a variety of animal cells, however, might now assign a new meaning to filopodia in protozoan parasites.

We here describe for the first time that emerging malaria gametes form membranous cell-to-cell connections upon gametocyte activation in the mosquito midgut. A detailed characterization of these filaments revealed that they have many of the features previously attributed to the long-distance filamentous cell connections of animal cells, which were referred to as nanotubes [reviewed in $1,2]$. In accordance with the hitherto existing description for nanotubes, the gamete-derived filaments are $\sim 200 \mathrm{~nm}$ in diameter, contain the F-actin isoform Pfact2, and represent close-ended membranous protrusions of the plasma membrane. Our observations on filament formation in live gametes suggest that they form actively following the stimulus for emergence from the enveloping erythrocyte and that they can be retracted, with multiple filaments protruding simultaneously from the same parasite.

Gametocyte activation is initiated and emergence is accompanied by a remarkable shedding of multiple membrane layers. On uptake by the female anopheline mosquito during the blood meal, activation of mature gametocytes is mediated by environmental factors of the mosquito midgut, including a drop in temperature and the presence of the mosquito-derived molecule xanthurenic acid [reviewed in 12,13]. Within $<10$ min the activated gametocytes have rounded up and egressed from the enveloping erythrocyte, thereby shedding both, EM and PVM. We showed that neither of these membranes contributes to malaria FiGs, but that FiGs represent F-actinpositive protrusion of the macrogamete surface. In this context, earlier studies showed that artificially induced cell extensions do not contain cytoskeletal elements, in contrast to naturally induced filaments, which always contain F-actin, and occasionally microtubules [reviewed in 1].

It remains to be answered, how gametocytes are able 
to form these extensive membrane protrusions within a few minutes after activation. It is tempting to speculate that internal membrane reservoirs in the gametocyte merge with the outer gamete plasma membrane to then develop into FiGs. The use of such membrane reservoirs has been described during tether formation in fibroblasts to buffer membrane tension [39]. In this context, it might be an intriguing idea that the subpellicular membrane complex provides this reservoir in gametocytes. The complex is formed in the developing gametocyte and consists of a subpellicular membrane vacuole underneath the plasmalemma, which is subtended by an array of longitudinally oriented microtubules [40]. The subpellicular membranes disappear shortly after gametocyte activation, coincident with filament formation. However, fusion of the subpellicular membrane with the gamete surface has never been observed.

We further demonstrated that the filamentous structures were not an artefact of long term in vitro culturing. FiGs were also formed by gametes of a newly established field isolate that was in culture for $<4$ months. Additionally, the filaments were generated following in vitro stimulation, but also when gametocytes were activated by endogenous factors within the midgut of the blood-feeding mosquito. Although the protrusions were formed by the gametes within minutes after activation, they remain present in zygotes after fertilization and can even be found in the spherical part of the retort stage during transformation into an ookinete.

Depending on the origin of the cell type, a number of different functions have been assigned to the membrane nanotubes of mammalian cells. Initial observations in Drosophila imaginal disc cells pointed to a role of the so-called cytonemes in long-range cell-cell communication by the result of receptor-ligand interactions [41, 42]. The formation of tubuvesicular extensions in neutrophils was attributed to neutrophil rolling in the context of inflammation and thrombosis, and additionally to executing long-range adhesion and binding of objects for phagocytosis [43-45]. The thread-like cytonemes of Bcells were considered to play a role in long-range communication and/or transportation of signaling molecules during an immune response [46]. Furthermore, vesicular cargo transport and an actomyosin-driven intercellular exchange of endocytic organelles were assigned to nanotubes of PC12 and macrophages as well as rat kidney cells $[3,9,47]$. Nanotube-like structures appear also to be used by infectious agents like HIV, bacteria or prions to spread between host cells $[6,9,48-50]$. Such diversity of assigned functions is consistent with the heterogeneity observed in nanotube properties. Nonetheless, the increasing number of cell types, in which nanotubes have been identified, leads to the assumption that these cell surface protuberances might represent ancient features of unicellular eukaryotes to explore the environment [42] in order to facilitate contact and establish communication with another cell.

Due to the facts that the malaria "nanotubes" exhibit adhesion proteins on their surface, which mediate cell binding, and that they further appear to be close ended without intercellular trafficking of cytoplasmic content, we propose that their functions lie in their adhesive properties. Gametes are the only stages within the malaria parasite life cycle that actually need to establish contact with another parasite in order to find a mating partner, followed by initiation of cell fusion and fertilization. We therefore hypothesize that the gamete-derived filaments are tools to locate, connect and pool gametocytes and gametes within the blood meal, which then results in clustering of sexual stage parasites. It has to be taken under consideration that under natural conditions $<100$ gametocytes are taken up in a blood meal by a mosquito, and that aggregation of gametocytes was proposed to facilitate fertilization [51].

In conclusion we report that $P$. falciparum sexual stages develop membranous cell extensions, which represent a novel type of cell protrusions widely used among different types of animal cells. We hypothesize that these membranous filaments mediate the contact between sexual stages in the mosquito midgut, thus promoting gamete aggregation. This work provides the initial identification and characterization of malaria "nanotubes" that can be used as a base for further functional evaluation of these fascinating cell protrusions.

\section{Materials and Methods}

\section{Gene IDs}

The following gene identifiers are assigned to the proteins investigated in this study: glurp, PF10_0344; msp1, PFI1475w; msp2, PFB0300c; Pf39, PF11_0098; Pfact2, PF14_0124; Pfatubulin II, PFD1050w; PfCCp1, PF14_0723; PfCCp2, PF14 0532; $P f C C p 3$, PF 14 0067; PfCCp4, PFI0185w; PfCCp5, PFA0445w; PfEXP-1, PF11_0224; PfFNPA, PF14_0491; PfPeg3, PFL0795c; $P f$ Peg4, PF10_0164; Pfs230, PFB0405w; Pfs 48/45, PF13_0247; $P f \mathrm{~s} 16, \mathrm{PFD} 0310 \mathrm{w} ; P f \mathrm{~s} 25$, PF10_0303; $P f \mathrm{~s} 28, \mathrm{PF} 10 \_0302$.

\section{Antibodies}

The following antibodies were used in this study: mouse polyclonal antisera against $P f C C p 1$ rp1, PfCCp2rp3, PfCCp3rp3, $P f$-CCp4rp1, $P f C \mathrm{Cp} 5 \mathrm{rp} 2, P f \mathrm{FNPArp} 2$ and $P f 39$ [21]; PfPeg3, $P f$ Peg4, and $P f \mathrm{~s} 16$ (kindly provided by P Alano, Rome); $P f \mathrm{~s} 230$ (against the $P f \mathrm{~s} 230$ immunogenic region C) [52, 53], and $P f \mathrm{~s} 48 / 45$ (American Type Culture Collection (ATCC)); mouse monoclonal antibodies against $\alpha$-tubulin, glycophorin A and band 3 (SigmaAldrich); rabbit antibodies against $\alpha$-tubulin II (ATCC) [35] and 
PfEXP-1 (kindly provided by J Przyborski, Marburg) [15] as well as $P f \mathrm{~s} 25$ and $P f \mathrm{~s} 28$ (ATCC). Mouse polyclonal antisera against $P f a c t 2$ were newly generated for this study (see below).

\section{Parasite lines}

The following $P$. falciparum parasite lines were used in this study: wild-type isolate NF54 (ATCC), wild-type strain 3D7 (ATCC), the gametocyte-less strain F12 (kindly provided by $\mathrm{P}$ Alano, Rome) and a recently obtained field isolate, 166MOA (see below); PfCCp1-KO [24]; $P f$ s48/45-KO (kindly provided by $\mathrm{R}$ Sauerwein, Nijmegen) [27]; $P f \mathrm{~s} 230-\mathrm{KO}$ d2 [25, 26] and the GFPexpressing parasite lines GFP-Pfnek-4 [28] and 5' CAM.GFP [29].

\section{Preparation of a parasite field isolate}

The $P$. falciparum isolate $166 \mathrm{MOA}$ was isolated from an asymptomatically infected individual from Lambaréné, Gabon. The study protocol was approved by the Ethics committee of the Albert Schweitzer Hospital. Approximately $5 \mathrm{ml}$ of anticoagulated blood was withdrawn and transported to the Medical Research Unit of the Albert Schweitzer Hospital at room temperature (RT). The sample was centrifuged at $1000 \times \mathrm{g}$ for $10 \mathrm{~min}$ and frozen in Glycerolyte solution as described [54]. The sample was subsequently stored at $-80{ }^{\circ} \mathrm{C}$ and transported to the Institute of Tropical Medicine at the University of Tübingen for in vitro culture (see below). Infection with $P$. falciparum was confirmed by gene amplification and sequencing of the merozoite surface proteins $m s p 1$ and $m s p 2$, as well as of the glutamate-rich protein glurp. The isolate was subsequently transferred to the Research Center for Infectious Diseases at the University of Würzburg.

\section{Parasite culture and membrane feeds}

Mature $P$. falciparum gametocytes were cultivated in vitro as described [55]. Gametocyte activation and gametogenesis was induced by incubating mature gametocyte cultures in emergence medium $(100 \mu \mathrm{M}$ xanthurenic acid, $1.67 \mathrm{mg} / \mathrm{ml}$ glucose; $8 \mathrm{mg} / \mathrm{ml}$ $\mathrm{NaCl}, 8 \mathrm{mM}$ Tris-Cl, pH 8.2) at RT [25]. Zygote formation was achieved by incubation of gametes overnight at RT in emergence medium. To investigate transmission stages in the mosquito midgut, mature gametocyte cultures were mixed with packed uninfected erythrocytes and centrifuged. The supernatant was replaced by normal human serum, mixed and membrane fed to Anopheles stephensi mosquitoes [56]. The mosquitoes were allowed to gorge for $5 \mathrm{~min}$. For live imaging, the midguts were immediately dissected and the blood meal analyzed via light microscopy using a Zeiss Axiovert200/Axiovision 4.3 microscope. For immunofluorescence assays, midguts were dissected $24 \mathrm{~h}$ post feeding, the midgut content smeared on a glass slide, and methanol-fixed. Immunofluorescence assays were carried out as described below.

\section{Treatment of in vitro cultures}

EM and PVM were removed prior to activation in emergence medium by incubating gametocytes with $0.05 \%$ of saponin in PBS for $5 \mathrm{~min}$ at RT and washed twice in PBS before activation in emergence medium. To investigate the possible influence of antibodies on filament formation, mature activated gametocyte cultures were incubated with 1:50 dilutions of antibodies against $P f \mathrm{~s} 230$ or $P f \mathrm{~s} 25$ in emergence medium for a period of $30 \mathrm{~min}$. Human serum of the culture medium was heat-inactivated in order to avoid complement-mediated lysis of gametes in antibody- treated cultures [20]. For control, cultures were incubated with a 1:50 dilution of neutral mouse serum or pure emergence medium. The cultures were subsequently fixed and filaments labeled by immunofluorescence assay as described below. Cultures incubated with $P f$ s230 antibodies were immunolabeled with antibodies against $P f \mathrm{~s} 25$ and vice versa. For the quantification of filamentforming gametes in cultures that exhibit different numbers of gametocytes, a mature gametocyte culture was diluted with cell culture medium $(1,1: 2,1: 10)$. Fresh erythrocytes were added to each $200 \mu \mathrm{l}$ sample to obtain a constant cell number of 11.1 $\times 10^{6}$ cells per sample. To investigate the possible influence of erythrocyte surface glycophorins on filament formation, mature gametocyte cultures were treated with $\alpha$-chymotrypsin (SigmaAldrich) prior to incubation in a final concentration of $2 \mathrm{mg} / \mathrm{ml}(20$ $\mathrm{mg} / \mathrm{ml}$ stock solution in $1 \mathrm{mM} \mathrm{HCl}$ and $2 \mathrm{mM} \mathrm{CaCl}_{2}$ ). Removal of glycophorins was confirmed by immunodetection of glycophorin A in lysates of treated versus untreated cultures via western blot analysis, using monoclonal antibodies directed against glycophorin A (Sigma-Aldrich; data not shown). To investigate the effect of cytochalasin B in FiG formation, the inhibitor (Sigma-Aldrich) was added to activated cultures at a final concentration of $50 \mathrm{nM}$ and incubated for $15 \mathrm{~min}$. This inhibitor concentration was shown to completely inhibit exflagellation in pre-tests (data not shown).

\section{Recombinant protein production and derivation of mouse antisera}

Recombinant protein for Pfact2 was expressed as a GSTtagged fusion protein using the pGEX 4T-1 vector (Amersham Biosciences). Cloning was mediated by the addition of Bam $\mathrm{HI}$ and NotI restriction sites to the ends of PCR-amplified gene fragments, using primers $5^{\prime}$-atggatcetatgtattacccatgcc-3' and 5'-tagcggecgettatgatcctcctatccatac-3'. Recombinant proteins were expressed in Escherichia coli BL21 (DE3) RIL cells according to the manufacturer's protocol (Stratagene). Recombinant proteins were isolated as inclusion bodies as described [20]. Specific immune sera were generated by the immunization of 6-week-old female NMRI mice (Charles River Laboratories, Sulzfeld) with $100 \mu \mathrm{g}$ recombinant protein emulsified in Freund's incomplete adjuvant (Sigma-Aldrich) followed by a boost after 4 weeks. Mice were anesthetized by intraperitoneal injection of a mixture of ketamine and xylazine according to the manufacturer's protocol (Sigma-Aldrich), and immune sera were collected 10 days after the second immunization via heart puncture. Housing and handling of the animals followed the guidelines of the animal welfare committee of the Government of Lower Franconia.

\section{Western blot analysis}

Pellets of saponin-lysed asexual parasites of wild-type isolate NF54 and the gametocyte-less strain F12, or Percoll gradientenriched NF54 gametocytes were resuspended in PBS and SDSPAGE loading buffer. In case of Pfact2 detection, an additional 5\% of an $8 \mathrm{M}$ urea solution was added to the samples. Parasite proteins were separated by SDS-PAGE electrophoresis and transferred to Hybond ECL nitrocellulose membrane (Amersham Biosciences) according to the manufacturer's instructions. Membranes were blocked for non-specific binding by incubation in Tris-buffered saline containing $5 \%$ of skim milk and $1 \%$ of BSA fraction $\mathrm{V}$, followed by immune recognition for $2 \mathrm{~h}$ at RT with mouse immune sera specific to Pfact2. After washing, membranes were incubated 
for $1 \mathrm{~h}$ at RT with an alkaline phosphatase-conjugated secondary antibody (Sigma-Aldrich) and developed in a solution of nitroblue tetrazolium and 5-bromo-4-chloro-3-indolyl phosphate (SigmaAldrich) for $5 \mathrm{~min}$.

\section{Indirect immunofluorescence assay}

Activated gametocyte cultures were air dried on glass slides and fixed for $10 \mathrm{~min}$ in $-80{ }^{\circ} \mathrm{C}$ methanol. For membrane permeabilization and blocking of nonspecific binding, fixed cells were successively incubated for $30 \mathrm{~min}$ in $0.01 \%$ saponin $/ 0.5 \% \mathrm{BSA} /$ PBS and 1\% neutral goat serum (Sigma-Aldrich) in PBS. Preparations were then incubated for $1.5 \mathrm{~h}$ at $37{ }^{\circ} \mathrm{C}$ with antibodies or immune sera generated against the indicated protein diluted in $0.01 \%$ saponin $/ 0.5 \%$ BSA/PBS. Binding of primary antibody was visualized using fluorescence-conjugated goat anti-mouse or anti-rabbit secondary antibodies (Alexa Fluor 488 or Fluor 596; Molecular Probes, Karlsruhe) diluted in $0.01 \%$ saponin $/ 0.5 \%$ BSA/PBS. For actin labeling, methanol-fixed cultures were treated with $0.1 \%$ Triton-X100/PBS and then incubated with TRITC-labeled phalloidin (Sigma-Aldrich). Counterstaining of erythrocytes was performed using $0.05 \%$ Evans Blue/PBS (Sigma-Aldrich) for $1 \mathrm{~min}$. Specimens were examined by confocal laser scanning microscopy using a Zeiss LSM 510 or using a Zeiss Axiolab microscope in combination with an Axiocam camera. Digital images were processed using Adobe Photoshop CS software. For quantitative evaluation of filament-positive cells over time, mature gametocyte cultures were fixed at 7, 15, 30, 60 and 120 min after activation, and labeled with antibodies against $P f \mathrm{~s} 230$. For quantitative evaluation of filaments in inhibitor-treated and gene-disruptant parasite lines, gametocyte cultures were fixed $30 \mathrm{~min}$ after activation and labeled with antibodies against $P f \mathrm{~s} 230$ or $P f_{\mathrm{s}} 25$. In all, 50 gametes were investigated for each condition, the number of filament-positive cells as well as the number of filaments per gamete counted and the length of each filament measured (using Zeiss LSM 510 software). Each condition was counted in triplicates and mean values were calculated. For quantitative evaluation of filament-forming gametes in antibody-treated cultures or cultures with different numbers of gametocytes, single settings were counted.

\section{Live imaging}

For live staining LysoSensorTM Green DND-189 (Molecular Probes) was added to activated gametocyte cultures in a final concentration of $4 \mu \mathrm{M}$. The cells were incubated for $5 \mathrm{~min}$, transferred to a glass slide and examined by confocal laser scanning microscopy (see above). For live video imaging, time course analyses were carried out using confocal laser scanning microscopy and converted into video files. For live imaging of $5^{\prime}$ CAM. GFP or GFP-Pfnek-4 parasites, mature gametocyte cultures were activated, immediately transferred to a glass slide and analyzed via fluorescence microscopy using a Zeiss LSM 510.

\section{Scanning electron microscopy}

Activated gametocyte cultures were transferred to cover slips, which were coated with $0.01 \%$ of Poly-L-Lysine (Sigma-Aldrich). Cells were allowed to settle for $1 \mathrm{~h}$ and then fixed with $6.25 \%$ glutaraldehyde/PBS overnight at $4{ }^{\circ} \mathrm{C}$. Fixed cells were washed twice with PBS, dehydrated in graded series of acetone and critical point dried using $\mathrm{CO}_{2}$, and specimens were sputtered with $30 \mathrm{~nm}$ gold. Images were taken with a Zeiss DSM 962 scanning electron microscope and processed using Adobe Photoshop CS software.

\section{Transmission electron microscopy}

Exflagellating gametocyte cultures were fixed in $1 \%$ of glutaraldehyde and $4 \%$ of paraformaldehyde in PBS for 2 days. Specimens were post fixed in $1 \%$ osmium tetroxide and $1.5 \%$ $\mathrm{K}_{3} \mathrm{Fe}(\mathrm{CN})_{6}$ in PBS for $2 \mathrm{~h}$ at $\mathrm{RT}$, followed by incubation in $0.5 \%$ uranyl acetate for $1 \mathrm{~h}$. The fixed cultures were dehydrated in increasing concentrations of ethanol and then incubated for $1 \mathrm{~h}$ in propylene oxide, followed by another incubation for $1 \mathrm{~h}$ in a 1:1 mixture of propylene oxide and Epon (Electron Microscopy Sciences). Specimens were subsequently embedded in Epon at $60^{\circ} \mathrm{C}$ for 2 days. Post staining of sections was done with $1 \%$ of uranyl acetate for $30 \mathrm{~min}$. Photographs were taken with a Zeiss EM10 transmission electron microscope and scanned images were processed using Adobe Photoshop CS software.

\section{Acknowledgments}

We thank Robert Sauerwein (Nijmegen Centre for Molecular Life Sciences) for providing the $P f_{S} 48 / 45$ gene-disruptant parasites and Pietro Alano (Istituto Superiore di Sanità Rome) for providing antibodies against $P f s 16, P f P e g 3$ and PfPeg4 as well as $P$. falciparum strain F12. We further thank Jude Przyborski (University of Marburg), Shruti Agarwal (University of Wuerzburg) and Florian Kohlhepp (University of Wuerzburg) for the immunofluorescence assays resulting in Figure 2B and Supplementary information, Figures S3B and S3D, as well as Andrea Kuehn (University of Wuerzburg) for western blotting on chymotrypsin-treated erythrocytes. We are grateful to Dan Davis (Imperial College London) and Georg Krohne (University of Wuerzburg) for helpful discussions and advice. This research was funded by an Emmy Noether grant and SFB479 grant from the Deutsche Forschungsgemeinschaft to GP, and by Public Health Service grants AI69314 and AI48826 from the National Institutes of Health to KCW. The work was additionally supported by the EU seventh framework program fund "MALSIG" to GP and CD and a doctorate short-term grant from the Deutscher Akademischer Austauschdienst to IR.

\section{References}

1 Davis DM, Sowinski S. Membrane nanotubes: dynamic longdistance connections between animal cells. Nat Rev Mol Cell Biol 2008; 9:431-436.

2 Gerdes HH, Carvalho RN. Intercellular transfer mediated by tunneling nanotubes. Curr Opin Cell Biol 2008; 20:470-475.

3 Rustom A, Saffrich R, Markovic I, Walther P, Gerdes HH. Nanotubular highways for intercellular organelle transport. Science 2004; 303:1007-1010.

4 Önfelt B, Nedvetzki S, Yanagi K, Davis DM. Cutting edge: membrane nanotubes connect immune cells. J Immunol 2004; 173:1511-1513.

5 Watkins SC, Salter RD. Functional connectivity between immune cells mediated by tunneling nanotubules. Immunity 2005; 23:309-318.

6 Sowinski S, Jolly C, Berninghausen O, et al. Membrane nanotubes physically connect $\mathrm{T}$ cells over long distances presenting a novel route for HIV-1 transmission. Nat Cell Biol 2008; 10:211-219. 
7 Arkwright PD, Luchetti F, Tour J, et al. Fas stimulation of T lymphocytes promotes rapid intercellular exchange of death signals via membrane nanotubes. Cell Res 2010; 20:72-88.

8 Fifadara $\mathrm{NH}$, Beer F, Ono S, Ono SJ. Interaction between activated chemokine receptor 1 and FcepsilonRI at membrane rafts promotes communication and F-actin-rich cytoneme extensions between mast cells. Int Immunol 2010; 22:113-128.

9 Önfelt B, Nedvetzki S, Benninger RK, et al. Structurally distinct membrane nanotubes between human macrophages support long-distance vesicular traffic or surfing of bacteria. $J$ Immunol 2006; 177:8476-8483.

10 Rustom, A. Hen or egg?:some thoughts on tunneling nanotubes. Ann N Y Acad Sci 2009; 1178:129-136.

11 Koyanagi M, Brandes RP, Haendeler J, Zeiher AM, Dimmeler S. Cell-to-cell connection of endothelial progenitor cells with cardiac myocytes by nanotubes: a novel mechanism for cell fate changes? Circ Res 2005; 96:1039-1041.

12 Pradel G. Proteins of the malaria parasite sexual stages: expression, function and potential for transmission blocking strategies. Parasitology 2007; 134:1911-1929.

13 Kuehn A, Pradel G. The coming-out of malaria gametocytes. J Biomed Biotechnol 2010; 2010:976827.

14 Ansorge I, Benting J, Bhakdi S, Lingelbach K. Protein sorting in Plasmodium falciparum-infected red blood cells permeabilized with the pore-forming protein streptolysin O. Biochem J 1996; 315:307-314.

15 Günther K, Tümmler M, Arnold HH, et al. An exported protein of Plasmodium falciparum is synthesized as an integral membrane protein. Mol Biochem Parasitol 1991; 46:149-157.

16 Baker DA, Daramola O, McCrossan MV, Harmer J, Targett GA. Subcellular localization of Pfs16, a Plasmodium falciparum gametocyte antigen. Parasitology 1994; 108:129-137.

17 Bruce MC, Carter RN, Nakamura K, Aikawa M, Carter R. Cellular location and temporal expression of the Plasmodium falciparum sexual stage antigen Pfs16. Mol Biochem Parasitol 1994; 65:11-22.

18 Silvestrini F, Bozdech Z, Lanfrancotti A, et al. Genome-wide identification of genes upregulated at the onset of gametocytogenesis in Plasmodium falciparum. Mol Biochem Parasitol 2005; 143:100-110.

19 Pradel G, Hayton K, Aravind L, et al. A multidomain adhesion protein family expressed in Plasmodium falciparum is essential for transmission to the mosquito. J Exp Med 2004; 199:1533-1544.

20 Scholz SM, Simon N, Lavazec C, Dude MA, Templeton TJ, Pradel G. PfCCp proteins of Plasmodium falciparum: gametocyte-specific expression and role in complement-mediated inhibition of exflagellation. Int J Parasitol 2008; 38:327-340.

21 Templeton TJ, Fujioka H, Aikawa M, Parker KC, Kaslow DC. Plasmodium falciparum Pfs40, renamed Pf39, is localized to an intracellular membrane-bound compartment and is not sexual stage-specific. Mol Biochem Parasitol 1997; 190:359365.

22 Duffy PE, Kaslow DC. A novel malaria protein, Pfs28, and Pfs 25 are genetically linked and synergistic as falciparum malaria transmission-blocking vaccines. Infect Immun 1997; 65:1109-1113.

23 Mair GR, Braks JA, Garver LS, et al. Regulation of sexual development of Plasmodium by translational repression. Sci- ence 2006; 313:667-669.

24 Simon N, Scholz SM, Moreira C, et al. Sexual stage adhesion proteins form multi-protein complexes in the malaria parasite Plasmodium falciparum. J Biol Chem 2009; 284:1453714546.

25 Eksi S, Stump A, Fanning SL, Shenouda MI, Fujioka H, Williamson KC. Targeting and sequestration of truncated Pfs230 in an intraerythrocytic compartment during Plasmodium falciparum gametocytogenesis. Mol Microbiol 2002; 44:15071516.

26 Eksi S, Czesny B, van Gemert GJ, Sauerwein RW, Eling W, Williamson KC. Malaria transmission-blocking antigen, Pfs230, mediates human red blood cell binding to exflagellating male parasites and oocyst production. Mol Microbiol 2006; 61:991-998.

27 van Dijk MR, Janse CJ, Thompson J, et al. A central role for $\mathrm{P} 48 / 45$ in malaria parasite male gamete fertility. Cell 2001; 104:153-164.

28 Reininger L, Billker O, Tewari R, et al. A NIMA-related protein kinase is essential for completion of the sexual cycle of malaria parasites. J Biol Chem 2005; 280:31957-31964.

29 Eksi S, Suri A, Williamson KC. Sex- and stage-specific reporter gene expression in Plasmodium falciparum. Mol Biochem Parasitol 2008; 160:148-151.

30 Cintra WM, De Souza W. Immunocytochemical localization of cytoskeletal proteins and electron microscopy of detergent extracted tachyzoites of Toxoplasma gondii. J Submicrosc Cytol 1985; 17:503-508.

31 Dobrowolski JM, Niesman IR, Sibley LD. Actin in the parasite Toxoplasma gondii is encoded by a single copy gene, ACT1 and exists primarily in a globular form. Cell Motil Cytoskeleton 1997; 37:253-262.

32 Wesseling JG, Smits MA, Schoenmakers JG. Extremely diverged actin proteins in Plasmodium falciparum. Mol Biochem Parasitol 1988; 30:143-153.

33 Wesseling JG, Snijders PJ, van Someren P, Jansen J, Smits MA, Schoenmakers JG. Stage-specific expression and genomic organization of the actin genes of the malaria parasite Plasmodium falciparum. Mol Biochem Parasitol 1989; 35:167-176.

34 Schüler H, Matuschewski K. Regulation of apicomplexan microfilament dynamics by a minimal set of actin-binding proteins. Traffic 2006; 7:1433-1439.

35 Rawlings DJ, Fujioka H, Fried M, Keister DB, Aikawa M, Kaslow DC. Alpha-tubulin II is a male-specific protein in Plasmodium falciparum. Mol Biochem Parasitol 1992; 56:239-250.

36 Macadam RF, Herbert WJ. Fine structural demonstration of cytoplasmic protrusions (filopodia) in Trypanosomes. Exp Parasitol 1970; 27:1-8.

37 Ito Y, Furuya M, Oka M, Osaki H, Aikawa M. Transmission and scanning electron microscopic studies of the micronemata of Trypanosoma gambiense. J Protozool 1981; 28:313-316.

38 Frevert U, Reinwald E. Formation of filopodia in Trypanosoma congolense by crosslinking the variant surface antigen. J Ultrastruct Mol Struct Res 1988; 99:124-136.

39 Raucher D, Sheetz MP. Characteristics of a membrane reservoir buffering membrane tension. Biophys $J$ 1999; 77:19922002. 
40 Sinden RE. Gametocytogenesis of Plasmodium falciparum in vitro: an electron microscopic study. Parasitology 1982; 84:111.

41 Ramirez-Weber FA, Kornberg TB. Cytonemes: cellular processes that project to the principal signaling center in Drosophila imaginal disks. Cell 1999; 97:599-607.

42 Ramirez-Weber FA, Kornberg TB. Signaling reaches to new dimensions in Drosophila imaginal discs. Cell 2000; 103:189192.

43 Schmidtke DW, Diamond SL. Direct observation of membrane tethers formed during neutrophil attachment to platelets or P-selectin under physiological flow. J Cell Biol 2000; 149:719-730.

44 Galkina SI, Sud'ina GF, Ullrich V. Inhibition of neutrophil spreading during adhesion to fibronectin reveals formation of long tubulovesicular cell extensions (cytonemes). Exp Cell Res 2001; 266:222-228.

45 Galkina SI, Molotkovsky JG, Ullrich V, Sud'ina GF. Scanning electron microscopy study of neutrophil membrane tubulovesicular extensions (cytonemes) and their role in anchoring, aggregation and phagocytosis. The effect of nitric oxide. Exp Cell Res 2005; 304:620-629.

46 Gupta N, DeFranco AL. Visualizing lipid raft dynamics and early signaling events during antigen receptor-mediated Blymphocyte activation. Mol Biol Cell 2003; 14:432-444.

47 Gurke S, Barroso JF, Hodneland E, Bukoreshtliev NV, Schlicker O, Gerdes HH. Tunneling nanotube (TNT)-like structures facilitate a constitutive, actomyosin-dependent exchange of endocytic organelles between normal rat kidney cells. Exp Cell Res 2008; 314:3669-3683.

48 Gousset K, Schiff E, Langevin C, et al. Prions hijack tunneling nanotubes for intercellular spread. Nat Cell Biol 2009; 11:328-336.

49 Eugenin EA, Gaskill PJ, Berman JW. Tunneling nanotubes
(TNT) are induced by HIV-infection of macrophages: a potential mechanism for intercellular HIV trafficking. Cell Immunol 2009; 254:142-148.

50 Eugenin EA, Gaskill PJ, Berman JW. Tunneling nanotubes (TNT): a potential mechanism for intercellular HIV trafficking. Commun Integr Biol 2009; 2:243-244.

51 Pichon G, Awono-Ambene HP, Robert V. High heterogeneity in the number of Plasmodium falciparum gametocytes in the bloodmeal of mosquitoes fed on the same host. Parasitology 2000; 121:115-120.

52 Williamson KC, Keister DB, Muratova O, Kaslow DC. Recombinant Pfs230, a Plasmodium falciparum gametocyte protein, induces antisera that reduce the infectivity of Plasmodium falciparum to mosquitoes. Mol Biochem Parasitol 1995; 75:33-42.

53 Bustamante PJ, Woodruff DC, Oh J, Keister DB, Muratova $\mathrm{O}$, Williamson KC. Differential ability of specific regions of Plasmodium falciparum sexual-stage antigen, Pfs230, to induce malaria transmission-blocking immunity. Parasite Immunol 2000; 22:373-380.

54 Haeggström M, Schlichtherle M. Freezing and thawing of asexual Plasmodium spp. In: Ljungström I, Perlmann H, Schlichtherle M and Wahlgren M, eds. Methods in Malaria Research (4 $4^{\text {th }}$ edition). Manassas, VA: Malaria Research and Reference Reagent Resource Center (MR4) American Type Culture Collection, 2004; 12-16.

55 Ifediba T, Vanderberg JP. Complete in vitro maturation of Plasmodium falciparum gametocytes. Nature 1981; 294:364366.

56 Bishop A, Gilchrist BM. Experiments upon the feeding of Aedes aegypti through animal membranes with a view to applying this method to chemotherapy of malaria. Parasitology 1946; 37:85-100.

(Supplementary information is linked to the online version of the paper on the Cell Research website.) 